\title{
STABILITY OF THE SOLUTIONS OF IMPULSIVE FUNCTIONAL-DIFFERENTIAL EQUATIONS BY LYAPUNOV'S DIRECT METHOD
}

\author{
D. D. BAINOV ${ }^{1}$ and I. M. STAMOVA ${ }^{2}$
}

(Received 27 May, 1997)

\begin{abstract}
We consider the stability of the zero solution of a system of impulsive functional-differential equations. By means of piecewise continuous functions, which are generalizations of classical Lyapunov functions, and using a technique due to Razumikhin, sufficient conditions are found for stability, uniform stability and asymptotical stability of the zero solution of these equations. Applications to impulsive population dynamics are also discussed.
\end{abstract}

\section{Introduction}

In the last few years impulsive differential equations have become the object of increasing investigation. A natural generalization of impulsive ordinary differential equations is impulsive functional-differential equations. In spite of the great possibilities for applications, the theory of these equations is developing rather slowly due to a series of difficulties of technical and theoretical character [1-3].

Effective application of these equations in mathematical modelling assumes knowledge of various criteria for stability of their solutions.

In this paper we investigate the stability of the zero solution of a system of impulsive functional-differential equations by virtue of piecewise continuous functions which are analogues of classical Lyapunov functions [4] in conjunction with a technique due to Razumikhin [5-7].

\footnotetext{
${ }^{1}$ Medical University of Sofia, P.O. Box 45, 1504 Sofia, Bulgaria.

${ }^{2}$ Technical University, Sliven, Bulgaria.

(C) Australian Mathematical Society 2001, Serial-fee code 0334-2700/01
} 


\section{Preliminary notes and definitions}

Let $\mathbb{R}^{n}$ be the $n$-dimensional Euclidean space with the norm $|\cdot|, \Omega$ a domain in $\mathbb{R}^{n}$ containing the origin and let $\mathbb{R}_{+}=[0, \infty)$. Also let $t_{0} \in \mathbb{R}$ and $\tau>0$.

We consider the system of impulsive functional-differential equations

$$
\begin{aligned}
\dot{x}(t) & =f\left(t, x_{t}\right), & & t>t_{0}, t \neq t_{k}, \\
\Delta x\left(t_{k}\right) & =x\left(t_{k}+0\right)-x\left(t_{k}-0\right)=I_{k}\left(x\left(t_{k}-0\right)\right), & & t_{k}>t_{0}, k=1,2, \ldots,
\end{aligned}
$$

where $f:\left(t_{0}, \infty\right) \times D \rightarrow \mathbb{R}^{n} ; D=\{\Phi:[-\tau, 0] \rightarrow \Omega, \Phi(t)$ is continuous everywhere except for a finite number of points $\tilde{t}$ at which $\Phi(\tilde{t}-0)$ and $\Phi(\tilde{t}+0)$ exist and $\Phi(\tilde{t}-0)=\Phi(\tilde{t})\} ; I_{k}: \Omega \rightarrow \mathbb{R}^{n}, k=1,2, \ldots ; t_{0}<t_{1}<t_{2}<\cdots$; $\lim _{k \rightarrow \infty} t_{k}=\infty$ and for $t>t_{0}, x_{t} \in D$ is defined by $x_{t}(s)=x(t+s),-\tau \leq s \leq 0$.

Let $\varphi_{0} \in D$. We denote by $x(t)=x\left(t ; t_{0}, \varphi_{0}\right)$ the solution of the system (1), which satisfies the initial conditions

$$
\begin{aligned}
& x\left(t ; t_{0}, \varphi_{0}\right)=\varphi_{0}\left(t-t_{0}\right), \quad t_{0}-\tau \leq t \leq t_{0}, \\
& x\left(t_{0}+0 ; t_{0}, \varphi_{0}\right)=\varphi_{0}(0) .
\end{aligned}
$$

The solution $x(t)=x\left(t ; t_{0}, \varphi_{0}\right)$ of the initial value problem (1), (2) is characterized by the following:

(a) For $t_{0}-\tau \leq t \leq t_{0}$ the solution $x(t)$ satisfies the initial conditions (2).

(b) For $t_{0}<t \leq t_{1}, x(t)$ coincides with the solution of the problem

$$
\begin{aligned}
& \dot{x}(t)=f\left(t, x_{t}\right), \quad t>t_{0}, \\
& x_{t_{0}}(s)=\varphi_{0}(s), \quad-\tau \leq s \leq 0 .
\end{aligned}
$$

At the moment $t=t_{1}$ the mapping point $\left(t, x\left(t ; t_{0}, \varphi_{0}\right)\right)$ moves "instantly" from the position $\left(t_{1}, x\left(t_{1} ; t_{0}, \varphi_{0}\right)\right)$ into the position $\left(t_{1}, x\left(t_{1} ; t_{0}, \varphi_{0}\right)+I_{1}\left(x\left(t_{1} ; t_{0}, \varphi_{0}\right)\right)\right)$.

(c) For $t_{1}<t \leq t_{2}$ the solution $x(t)$ coincides with the solution of the problem

$$
\begin{aligned}
\dot{y}(t) & =f\left(t, y_{t}\right), & & t>t_{1}, \\
y_{t_{1}} & =\varphi_{1}, & & \varphi_{1} \in D,
\end{aligned}
$$

where

$$
\varphi_{1}\left(t-t_{1}\right)= \begin{cases}\varphi_{0}\left(t-t_{1}\right) & \text { for } t \in\left[t_{0}-\tau, t_{0}\right] \cap\left[t_{1}-\tau, t_{1}\right] \\ x\left(t ; t_{0}, \varphi_{0}\right) & \text { for } t \in\left(t_{0}, t_{1}\right), \\ x\left(t ; t_{0}, \varphi_{0}\right)+I_{1}\left(x\left(t ; t_{0}, \varphi_{0}\right)\right) & \text { for } t=t_{1}\end{cases}
$$

At the moment $t=t_{2}$ the mapping point $\left(t, x\left(t ; t_{0}, \varphi_{0}\right)\right)$ moves "instantly" and so on. 
The solution $x(t)=x\left(t ; t_{0}, \varphi_{0}\right)$ of the initial value problem (1), (2) is a piecewise continuous function for $t>t_{0}$ with points of discontinuity of the first kind $t_{k}, k=$ $1,2, \ldots$, where it is continuous from the left.

We also introduce the following notation:

$$
\begin{gathered}
I=\left[t_{0}-\tau, \infty\right) ; \quad I_{0}=\left[t_{0}, \infty\right) ; \\
G_{k}=\left\{(t, x) \in I_{0} \times \Omega: t_{k-1}<t<t_{k}\right\}, \quad k=1,2, \ldots ; \quad G=\bigcup_{k=1}^{\infty} G_{k} ; \\
\|\Phi\|=\sup _{s \in[-\tau, 0]}|\Phi(s)| \text { is the norm of the function } \Phi \in D .
\end{gathered}
$$

Let $J \subset \mathbb{R}$. We define the following classes of functions:

$P C\left[J, \mathbb{R}^{n}\right]=\left\{\sigma: J \rightarrow \mathbb{R}^{n}: \sigma(t)\right.$ is continuous everywhere except for some points $t_{k}$ at which $\sigma\left(t_{k}-0\right)$ and $\sigma\left(t_{k}+0\right)$ exist and $\left.\sigma\left(t_{k}-0\right)=\sigma\left(t_{k}\right)\right\}$

$P C^{1}\left[J, \mathbb{R}^{n}\right]=\left\{\sigma \in P C\left[J, \mathbb{R}^{n}\right]: \sigma(t)\right.$ is continuously differentiable everywhere except for some points $t_{k}$ at which $\dot{\sigma}\left(t_{k}-0\right)$ and $\dot{\sigma}\left(t_{k}+0\right)$ exist and $\dot{\sigma}\left(t_{k}-0\right)=\dot{\sigma}\left(t_{k}\right)$ \};

$\mathscr{K}=\left\{a \in C\left[\mathbb{R}_{+}, \mathbb{R}_{+}\right]: a(u)\right.$ is a strictly increasing function with respect to $u$ and $a(0)=0\}$.

We also introduce the following conditions:

H1. $f \in C\left[I_{0} \times D, \mathbb{R}^{n}\right]$.

H2. $f(t, 0)=0, t \in I_{0}$.

H3. The function $f$ satisfies Lischitz's condition with respect to its second argument in $I_{0} \times D$ uniformly with respect to $t \in I_{0}$.

H4. $I_{k} \in C\left[\Omega, \mathbb{R}^{n}\right], k=1,2, \ldots$

H5. $I_{k}(0)=0, k=1,2, \ldots$.

H6. The functions $\left(I+I_{k}\right): \Omega \rightarrow \Omega, k=1,2, \ldots$, where $I$ is the identity mapping in $\Omega$.

H7. $t_{0}<t_{1}<t_{2}<\cdots$.

H8. $\lim _{k \rightarrow \infty} t_{k}=\infty$.

DEFINITION 1. The zero solution $x(t) \equiv 0$ of the system (1) is said to be:

(a) Stable if

$$
\begin{gathered}
\left(\forall t_{0} \in \mathbb{R}\right)(\forall \varepsilon>0)\left(\exists \delta=\delta\left(t_{0}, \varepsilon\right)>0\right) \\
\left(\forall \varphi_{0} \in D:\left\|\varphi_{0}\right\|<\delta\right)\left(\forall t>t_{0}\right):\left|x\left(t ; t_{0}, \varphi_{0}\right)\right|<\varepsilon .
\end{gathered}
$$

(b) Uniformly stable if the number $\delta$ from (a) can be chosen independently of $t_{0} \in \mathbb{R}$. 
(c) Uniformly attractive if

$$
\begin{gathered}
(\exists \lambda>0)(\forall \varepsilon>0)(\exists T=T(\varepsilon)>0)\left(\forall t_{0} \in \mathbb{R}\right) \\
\left(\forall \varphi_{0} \in D:\left\|\varphi_{0}\right\|<\lambda\right)\left(\forall t \geq t_{0}+T\right):\left|x\left(t ; t_{0}, \varphi_{0}\right)\right|<\varepsilon .
\end{gathered}
$$

(d) Uniformly asymptotically stable if it is uniformly stable and uniformly attractive.

In the following considerations we shall use a class of piecewise continuous auxiliary functions, which are analogues of the Lyapunov functions [4].

DefinItion 2. We say that the function $V: I_{0} \times \Omega \rightarrow \mathbb{R}_{+}$belongs to the class $\mathscr{V}$ if:

1. $V \in C\left[G, \mathbb{R}_{+}\right]$and $V(t, 0)=0$ for $t \in I_{0}$.

2. The function $V$ satisfies Lipschitz's condition with respect to its second argument $x \in \Omega$ on each of the subsets $G_{k}, k=1,2, \ldots$

3. For each $k=1,2, \ldots$ and all $x \in \Omega$ there exist the finite limits

$$
V\left(t_{k}-0, x\right)=\lim _{\substack{t \rightarrow t_{k} \\ i<t_{k}}} V(t, x), \quad V\left(t_{k}+0, x\right)=\lim _{\substack{t \rightarrow t_{k} \\ i>t_{k}}} V(t, x)
$$

4. The equality $V\left(t_{k}-0, x\right)=V\left(t_{k}, x\right), x \in \mathbb{R}^{n}, k=1,2, \ldots$, holds true.

Further on, we shall use the functional class

$$
\begin{aligned}
\Omega_{1}=\{x & \in P C\left[I_{0}, \mathbb{R}^{n}\right]: V(s, x(s)) \leq V(t, x(t)), t-\tau \leq s \leq t, \\
& \left.t \geq t_{0}, V \in \mathcal{Y}\right\} .
\end{aligned}
$$

Let $V \in \mathscr{Y}, x \in P C\left[I_{0}, \mathbb{R}^{n}\right], t \neq t_{k}, k=1,2, \ldots$ We define the function

$$
D_{-} V(t, x(t))=\lim _{h \rightarrow 0^{-}} \frac{1}{h}\left[V\left(t+h, x(t)+h f\left(t, x(t), x_{t}\right)\right)-V(t, x(t))\right] .
$$

When proving the main results of the paper we shall use the following assertions.

LEMMA 1 ([3]). Let the following conditions hold:

1. Conditions $\mathrm{H1}-\mathrm{H} 8$ are fulfilled.

2. The solution $x(t)=x\left(t ; t_{0}, \varphi_{0}\right)$ of the initial value problem (1), (2) is such that $x \in P C\left[I, \mathbb{R}^{n}\right] \cap P C^{1}\left[I_{0}, \mathbb{R}^{n}\right]$.

3. $g \in P C\left[I_{0} \times \mathbb{R}_{+}, \mathbb{R}\right] \cap P C^{1}\left[I_{0} \times \mathbb{R}_{+}, \mathbb{R}\right]$ and $g(t, 0)=0$ for $t \in I_{0}$.

4. $B_{k} \in C\left[\mathbb{R}_{+}, \mathbb{R}\right], k=1,2, \ldots$

5. The maximal solution $r\left(t ; t_{0}, u_{0}\right)$ of the impulsive problem

$$
\begin{array}{ll}
\dot{u}(t)=g(t, u(t)), & t>t_{0}, t \neq t_{k}, k=1,2, \ldots, \\
u\left(t_{0}+0\right)=u_{0}, & \\
\Delta u\left(t_{k}\right)=B_{k}\left(u\left(t_{k}\right)\right), \quad t_{k}>t_{0}, k=1,2, \ldots,
\end{array}
$$

is defined on the interval $I_{0}$. 
6. The functions $\psi_{k}: \mathbb{R}_{+} \rightarrow \mathbb{B}, \psi_{k}=u+B_{k}(u), k=1,2, \ldots$, are nondecreasing with respect to $u$.

7. The function $V \in \mathscr{V}$ is such that

$$
\begin{aligned}
V\left(t_{0}+0, \varphi_{0}(0)\right) & \leq u_{0}, & & \\
D_{-} V(t, x(t)) & \leq g(t, V(t, x(t))), & & t \in I_{0}, t \neq t_{k}, x \in \Omega_{1}, \\
V\left(t_{k}+0, x\left(t_{k}\right)+I_{k}\left(x\left(t_{k}\right)\right)\right) & \leq \psi_{k}\left(V\left(t_{k}, x\left(t_{k}\right)\right)\right), & & k=1,2, \ldots .
\end{aligned}
$$

Then $V\left(t, x\left(t ; t_{0}, \varphi_{0}\right)\right) \leq r\left(t ; t_{0}, u_{0}\right), t \in I_{0}$.

COROLLARY 1. Let the following conditions hold:

1. Conditions $\mathrm{H} 1-\mathrm{H} 8$ are fulfilled.

2. Condition 2 of Lemma 1 holds true.

3. The function $V \in \mathscr{V}$ is such that

$$
\begin{aligned}
D_{-} V(t, x(t)) & \leq 0, & & t \in I_{0}, t \neq t_{k}, x \in \Omega_{1}, \\
V\left(t_{k}+0, x\left(t_{k}\right)+I_{k}\left(x\left(t_{k}\right)\right)\right) & \leq V\left(t_{k}, x\left(t_{k}\right)\right), & & k=1,2, \ldots .
\end{aligned}
$$

Then $V\left(t, x\left(t ; t_{0}, \varphi_{0}\right)\right) \leq V\left(t_{0}+0, \varphi_{0}(0)\right), t \in I_{0}$.

\section{Main results}

THEOREM 1. Let the following conditions hold:

1. Conditions $\mathrm{H} 1-\mathrm{H} 8$ are fulfilled.

2. The functions $V \in \mathscr{V}$ and $a \in \mathscr{K}$ are such that

$$
a(|x|) \leq V(t, x), \quad(t, x) \in I_{0} \times \Omega .
$$

3. The inequalities

$$
\begin{aligned}
D_{-} V(t, x(t)) & \leq 0, & & t \in I_{0}, t \neq t_{k}, k=1,2, \ldots, \\
V\left(t+0, x(t)+I_{k}(x(t))\right) & \leq V(t, x(t)), & & t=t_{k}, k=1,2, \ldots
\end{aligned}
$$

hold true for $t \in I_{0}, x \in \Omega_{1}, V \in \mathscr{Y}$.

Then the zero solution of the system (1) is stable.

PROOF. Let $\varepsilon>0$. It follows from the condition $V\left(t_{0}, 0\right)=0$ and the properties of the function $V\left(t_{0}, x\right)$ that there exists a constant $\delta=\delta\left(t_{0}, \varepsilon\right)>0$ such that if $|x|<\delta$ then $\sup _{|x|<\delta} V\left(t_{0}+0, x\right)<a(\varepsilon)$. 
Let $\varphi_{0} \in D:\left\|\varphi_{0}\right\|<\delta$. Then $\left|\varphi_{0}(0)\right| \leq\left\|\varphi_{0}\right\|<\delta$ and therefore

$$
V\left(t_{0}+0, \varphi_{0}(0)\right)<a(\varepsilon)
$$

Let $x(t)=x\left(t ; t_{0}, \varphi_{0}\right)$ be the solution of the problem (1), (2). Since all the conditions of Corollary 1 are fulfilled, we have

$$
V\left(t, x\left(t ; t_{0}, \varphi_{0}\right)\right) \leq V\left(t_{0}+0, \varphi_{0}(0)\right), \quad t \in I_{0} .
$$

There follow from (3), (6) and (7) the inequalities

$$
a\left(\left|x\left(t ; t_{0}, \varphi_{0}\right)\right|\right) \leq V\left(t, x\left(t ; t_{0}, \varphi_{0}\right)\right) \leq V\left(t_{0}+0, \varphi_{0}(0)\right)<a(\varepsilon),
$$

whence we obtain that $\left|x\left(t ; t_{0}, \varphi_{0}\right)\right|<\varepsilon$ for $t>t_{0}$. This implies that the solution $x(t) \equiv 0$ of the system (1) is stable.

THEOREM 2. Let the conditions of Theorem 1 be fulfilled, and suppose that there exists a function $b \in \mathscr{K}$, such that

$$
V(t, x) \leq b(|x|), \quad(t, x) \in I_{0} \times \Omega .
$$

Then the zero solution of the system (1) is uniformly stable.

PROOF. Let $\varepsilon>0$ be chosen. We get $\delta=\delta(\varepsilon)>0$ such that $b(\delta)<a(\varepsilon)$. Let $\varphi_{0} \in D:\left\|\varphi_{0}\right\|<\delta$ and $x(t)=x\left(t ; t_{0}, \varphi_{0}\right)$ be the solution of the initial value problem (1), (2).

As in Theorem 1 we prove that

$$
a\left(\left|x\left(t ; t_{0}, \varphi_{0}\right)\right|\right) \leq V\left(t, x\left(t ; t_{0}, \varphi_{0}\right)\right) \leq V\left(t_{0}+0, \varphi_{0}(0)\right) .
$$

The above inequalities and (8) imply the inequalities

$$
a\left(\left|x\left(t ; t_{0}, \varphi_{0}\right)\right|\right) \leq V\left(t_{0}+0, \varphi_{0}(0)\right) \leq b\left(\left|\varphi_{0}(0)\right|\right) \leq b\left(\left\|\varphi_{0}\right\|\right)<b(\delta)<a(\varepsilon),
$$

whence it follows that $\left|x\left(t ; t_{0}, \varphi_{0}\right)\right|<\varepsilon$ for $t \in I_{0}$. This proves the uniform stability of the zero solution of the system (1).

THEOREM 3. Let the following conditions hold:

1. Conditions $\mathrm{H} 1-\mathrm{H} 8$ are fulfilled.

2. The functions $V \in \mathscr{V}$ and $a, b \in \mathscr{K}$ are such that

$$
a(|x|) \leq V(t, x) \leq b(|x|), \quad(t, x) \in I_{0} \times \Omega .
$$


3. The inequalities

$$
\begin{aligned}
D_{-} V(t, x(t)) & \leq-c(|x(t)|), & & t \in I_{0}, t \neq t_{k}, k=1,2, \ldots, \\
V\left(t+0, x(t)+I_{k}(x(t))\right) & \leq V(t, x(t)), & & t \in I_{0}, t=t_{k}, k=1,2, \ldots
\end{aligned}
$$

hold true for $x \in \Omega_{1}, V \in \mathscr{V}, c \in \mathscr{K}$.

Then the zero solution of the system (1) is uniformly asymptotically stable.

PRoOF. 1. Let $\alpha=$ const $>0:\{x \in \Omega:|x| \leq \alpha\} \subset \Omega$. For each $t \in I_{0}$ we denote

$$
V_{t, \alpha}^{-1}=\{x \in \Omega: V(t+0, x) \leq a(\alpha)\} .
$$

From (9) we deduce

$$
V_{t, \alpha}^{-1} \subset\{x \in \Omega:|x| \leq \alpha\} \subset \Omega .
$$

It follows from conditions 2 and 3 of Theorem 3 that for each function $\varphi_{0} \in D$ : $\varphi_{0}(0) \in V_{t_{0}, \alpha}^{-1}$ we have $x\left(t ; t_{0}, \varphi_{0}\right) \in V_{t, \alpha}^{-1}, \quad t>t_{0}$.

Let $\varepsilon>0$ be chosen. We get $\eta=\eta(\varepsilon)>0$ such that $b(\eta)<a(\varepsilon)$, and let $T>b(\alpha) / c(\eta)$.

If we suppose that for each $t \in\left[t_{0}, t_{0}+T\right]$ the inequality $\left|x\left(t ; t_{0}, \varphi_{0}\right)\right| \geq \eta$ is fulfilled, then (10) and (11) imply the inequalities

$$
\begin{aligned}
V\left(t_{0}+T, x\left(t_{0}+T ; t_{0}, \varphi_{0}\right)\right) & =V\left(t_{0}+0, \varphi_{0}(0)\right)-\int_{\iota_{0}}^{t_{0}+T} c\left(\left|x\left(s ; t_{0}, \varphi_{0}\right)\right|\right) d s \\
& \leq b(\alpha)-c(\eta) T<0,
\end{aligned}
$$

which contradicts (9). Therefore, there exists $t_{1}^{*} \in\left[t_{0}, t_{0}+T\right]$ such that $\left|x\left(t_{1}^{*} ; t_{0}, \varphi_{0}\right)\right|<$ $\eta$. Thus we obtain from (9), (10) and (11) that for $t \geq t_{1}^{*}$ (and therefore for $t \geq t_{0}+T$ ) the inequalities

$$
\begin{aligned}
a\left(\left|x\left(t ; t_{0}, \varphi_{0}\right)\right|\right) & \leq V\left(t, x\left(t ; t_{0}, \varphi_{0}\right)\right) \leq V\left(t_{1}^{*}, x\left(t_{1}^{*} ; t_{0}, \varphi_{0}\right)\right) \\
& \leq b\left(\left|x\left(t_{1}^{*} ; t_{0}, \varphi_{0}\right)\right|\right)<b(\eta)<a(\varepsilon)
\end{aligned}
$$

are valid. Therefore $\left|x\left(t ; t_{0}, \varphi_{0}\right)\right|<\varepsilon$ for $t \geq t_{0}+T$.

2. Let $\lambda=$ const $>0$ be such that $b(\lambda) \leq a(\alpha)$. Then, if $\varphi_{0} \in D:\left\|\varphi_{0}\right\|<\lambda$, there follow from (9) the inequalities

$$
V\left(t_{0}+0, \varphi_{0}(0)\right) \leq b\left(\left|\varphi_{0}(0)\right|\right) \leq b\left(\left\|\varphi_{0}\right\|\right)<b(\lambda) \leq a(\alpha),
$$

which show that $\varphi_{0} \in D: \varphi_{0}(0) \in V_{t_{0}, \alpha}^{-1}$.

It follows from the two points above that the zero solution of the system (1) is uniformly attractive and since it is uniformly stable according to Theorem 2 , then the solution is uniformly asymptotically stable. 
COROLLARY 2. If the third condition (10) in Theorem 3 is replaced by

$$
\text { D_ } V(t, x(t)) \leq-c V(t, x(t)) \text {, }
$$

for $t \in I_{0}, t \neq t_{k}, k=1,2, \ldots, x \in \Omega_{1}, c=$ const $>0$, then the zero solution of the system (1) is uniformly asymptotically stable.

PROOF. The proof of Corollary 2 is analogous to the proof of Theorem 3. It uses the inequality

$$
V\left(t, x\left(t ; t_{0}, \varphi_{0}\right)\right) \leq V\left(t_{0}+0, \varphi_{0}(0)\right) e^{-c\left(t-t_{0}\right)},
$$

which follows from (11) and (12).

In fact, let $\alpha=$ const $>0:\{x \in \Omega:|x| \leq \alpha\} \subset \Omega$. We choose $\lambda>0$ such that $b(\lambda)<a(\alpha)$.

Let $\varepsilon>0$ and $T>(1 / c) \ln (a(\alpha) / a(\varepsilon))$. Then for $\varphi_{0} \in D:\left\|\varphi_{0}\right\|<\lambda$ and $t \geq t_{0}+T$ the inequalities

$$
V\left(t, x\left(t ; t_{0}, \varphi_{0}\right)\right) \leq V\left(t_{0}+0, \varphi_{0}(0)\right) e^{-c\left(t-t_{0}\right)}<a(\varepsilon), \quad t \geq t_{0}+T
$$

hold true.

The last inequality and (9) imply that the zero solution of the system (1) is uniformly attractive.

\section{Applications in impulsive population dynamics}

Some of the results obtained in the present work will be applied below in studying mathematical models in impulsive population dynamics.

4.1. We consider the system

$$
\begin{aligned}
\dot{x}(t) & =B(t) x(t-h(t)), & & t>0, t \neq t_{k}, k=1,2, \ldots, \\
\Delta x\left(t_{k}\right) & =C_{k} x\left(t_{k}\right), & & k=1,2, \ldots,
\end{aligned}
$$

where $x \in \mathbb{R}^{n} ; B(t)$ is a diagonal $n \times n$-matrix valued function; $h \in C\left[\mathbb{R}_{+},[0, \tau]\right]$; $C_{k}=\operatorname{diag}\left(c_{1 k}, \ldots, c_{n k}\right), c_{i k} \leq-2 ; 0<t_{1}<t_{2}<\cdots$ and $\lim _{k \rightarrow \infty} t_{k}=\infty$.

Let $V(t, x)=x^{2} / 2$. Then

$$
\Omega_{1}=\left\{x \in P C\left[\mathbb{R}_{+}, \mathbb{R}^{n}\right]: x^{2}(s) \leq x^{2}(t), t-\tau \leq s \leq t, t \geq 0\right\} .
$$

If $\varphi_{0} \in P C\left[[-\tau, 0], \mathbb{R}^{n}\right]$ and $x\left(t ; \varphi_{0}\right)$ is the solution of the system (13) for which

$$
x\left(t ; \varphi_{0}\right)=\varphi_{0}(t), \quad t \in[-\tau, 0],
$$


we have for $t \in \mathbb{R}_{+}$and $x \in \Omega_{1}$ that

$$
D_{-} V(t, x(t))=x(t) \dot{x}(t)=B(t) x(t) x(t-h(t)) \leq B(t) x^{2}(t), \quad t \neq t_{k}, k=1,2, \ldots
$$

and

$$
V\left(t_{k}+0, x\left(t_{k}\right)+C_{k} x\left(t_{k}\right)\right)=\left[x\left(t_{k}\right)+C_{k} x\left(t_{k}\right)\right]^{2} / 2 \leq V\left(t_{k}, x\left(t_{k}\right)\right), \quad k=1,2, \ldots
$$

If the elements of $B(t)$ are nonpositive for $t \in \mathbb{B}_{+}$, then $D_{-} V(t, x(t)) \leq 0$ and the zero solution of the system (13) is uniformly stable according to Theorem 2 .

If $B(t)=\operatorname{diag}\left(b_{1}(t), \ldots, b_{n}(t)\right)$ and $b_{k}(t) \leq-\gamma_{k}<0, k=1,2, \ldots, n$, then the conditions of Theorem 3 are fulfilled and the zero solution of the system (13) is uniformly asymptotically stable.

4.2. Let us consider the equation

$$
\begin{aligned}
\dot{x}(t) & =r x(t)\left[1-\frac{x(t-\tau)}{K}\right], & & t>0, t \neq t_{k}, \\
\Delta x\left(t_{k}\right) & =\alpha_{k} x\left(t_{k}\right), & & t_{k}>0, k=1,2, \ldots,
\end{aligned}
$$

where $r>0, K>0, \tau>0, \alpha_{k}=$ const $>0, k=1,2, \ldots ; 0<t_{1}<t_{2}<\cdots$ and $\lim _{k \rightarrow \infty} t_{k}=\infty$. Let $x(s)=\varphi_{0}(s) \geq 0, s \in[-\tau, 0], \varphi_{0}(0)>0$ and $\varphi_{0}(s) \in D$.

We consider the function

$$
V(t, x)=(1-x / K)^{2}
$$

Then

$$
\begin{aligned}
\Omega_{1}= & \left\{x \in P C\left[\mathbb{B}_{+},(0, \infty)\right]:(1-x(s) / K)^{2} \leq(1-x(t) / K)^{2},\right. \\
& t-\tau \leq s \leq t, t \geq 0\} .
\end{aligned}
$$

We have for $t \geq 0, t \neq t_{k}$ and $x \in \Omega_{1}$ that

$$
\begin{aligned}
D_{-} V(t, x(t)) & =-\frac{2 r}{K}\left(1-\frac{x(t)}{K}\right) x(t)\left(1-\frac{x(t-\tau)}{K}\right) \\
& \leq-\frac{2 r}{K} x(t) V(t, x(t)) \leq 0 .
\end{aligned}
$$

Moreover, for $k=1,2, \ldots$,

$$
V\left(t_{k}+0, x\left(t_{k}\right)+\alpha_{k} x\left(t_{k}\right)\right)=\left[1-\left(1+\alpha_{k}\right) x\left(t_{k}\right) / K\right]^{2}<V\left(t_{k}, x\left(t_{k}\right)\right) .
$$

Since the conditions of Theorem 2 are met, then the zero solution of the system (14) is uniformly stable.

\subsection{Consider the equation}

$$
\begin{aligned}
\dot{x}(t) & =r x(t)\left[\frac{K-x(t-\tau)}{1+\operatorname{crx}(t-\tau)}\right], & & t>0, t \neq t_{k}, \\
\Delta x\left(t_{k}\right) & =\alpha_{k} x\left(t_{k}\right), & & t_{k}>0, k=1,2, \ldots,
\end{aligned}
$$


where $c>0$.

If the function $V(t, x)=((K-x) /(1+c r x))^{2}$, then

$$
\Omega_{1}=\left\{x \in P C\left[\mathbb{R}_{+},(0, \infty)\right]:\left[\frac{K-x(s)}{1+\operatorname{crx}(s)}\right]^{2} \leq\left[\frac{K-x(t)}{1+\operatorname{crx}(t)}\right]^{2}, t-\tau \leq s \leq t\right\} .
$$

For $t>0$ and $x \in \Omega_{1}$ the inequalities

$$
\begin{aligned}
D_{-} V(t, x(t)) & =-\frac{2 r(1+c r K)}{(1+c r x(t))^{2}} x(t)\left[\frac{K-x(t)}{1+c r x(t)}\right]\left[\frac{K-x(t-\tau)}{1+\operatorname{crx}(t-\tau)}\right] \\
& \leq-\frac{2 r(1+c r K)}{[1+\operatorname{cr} x(t)]^{4}} x(t)[K-x(t)]^{2} \leq 0, \quad t \neq t_{k}
\end{aligned}
$$

hold true, and, for $k=1,2, \ldots$,

$$
V\left(t_{k}+0, x\left(t_{k}\right)+\alpha_{k} x\left(t_{k}\right)\right)=\left[\frac{K-\left(1+\alpha_{k}\right) x\left(t_{k}\right)}{1+\operatorname{cr}\left(1+\alpha_{k}\right) x\left(t_{k}\right)}\right]^{2} \leq V\left(t_{k}, x\left(t_{k}\right)\right),
$$

and by virtue of Theorem 2 the zero solution of the system (15) is uniformly stable.

\section{Acknowledgement}

The present investigation was supported by the Bulgarian Ministry of Education, Science and Technologies under Grant MM-422.

\section{References}

[1] D. D. Bainov, V. C. Covachev and I. M. Stamova, "Estimates of the solutions of impulsive quasilinear functional differential equations", Ann. Fac. Sci. de Toulouse 2 (1991) 149-161.

[2] D. D. Bainov, V. C. Covachev and I. M. Stamova, "Stability under persistent disturbances of impulsive differential-difference equations of neutral type", J. Math. Anal. Appl. 187 (1994) 790-808.

[3] D. D. Bainov, G. K. Kulev and I. M. Stamova, "Global stability of the solutions of impulsive differential-difference equations", SUT J. of Math. 31 (1995) 55-71.

[4] D. D. Bainov and P. S. Simeonov, Systems with impulse effect: stability, theory and applications (Ellis Horwood, Chichester, 1989).

[5] V. Lakshmikantham, "Functional differential systems and extension of Lyapunov's method", J. Math. Anal. Appl. 8 (1964) 392-405.

[6] V. Lakshmikantham, S. Leela and A. A. Martynyuk, Stability analysis of nonlinear systems (Marcel Dekker, New York, 1989).

[7] B. S. Razumikhin, Stability of systems with retardation, in Russian (Nauka, Moscow, 1988). 